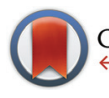

CrossMark $\leftarrow$ click for updates

Cite this: Dalton Trans., 2016, 45, 4132

Received 17th August 2015, Accepted 11th November 2015

DOI: $10.1039 / \mathrm{c} 5 \mathrm{dt} 03178 \mathrm{~h}$

www.rsc.org/dalton

\section{Postsynthetic bromination of UiO-66 analogues: altering linker flexibility and mechanical compliance $\uparrow$}

\author{
Ross J. Marshall, ${ }^{a}$ Tom Richards, ${ }^{b}$ Claire L. Hobday, ${ }^{c}$ Colin F. Murphie, ${ }^{a}$ \\ Claire Wilson, ${ }^{a}$ Stephen A. Moggach, ${ }^{c}$ Thomas D. Bennett*b and Ross S. Forgan ${ }^{\star a}$
}

\begin{abstract}
A new member of the UiO-66 series of zirconium metal-organic frameworks (MOFs) is reported, and the postsynthetic bromination of its integral alkene moeities in a single-crystal to single-crystal manner is fully characterised. Nanoindentation is used to probe the bromination of unsaturated carbon-carbon bonds, in it and an analogous $\mathrm{Zr}$ MOF, which leads to more compliant materials with lower elastic moduli.
\end{abstract}

Metal-organic frameworks (MOFs) are network materials comprised of metal ions or clusters connected by organic linkers into multidimensional structures which often exhibit permanent porosity. ${ }^{1}$ They have received considerable interest over the last 15 years as a result of their significant potential for application in, for example, gas storage and separation, ${ }^{2}$ catalysis, ${ }^{3}$ sensing, ${ }^{4}$ and drug delivery. ${ }^{5}$ The presence of dynamic components, or intrinsic framework flexibility, ${ }^{6}$ leads to stimuli-responsive materials ${ }^{7}$ which undergo structural changes under applied pressure, temperature or light. ${ }^{8}$ Flexibility is often used non-specifically or interchangeably with mechanical compliance, framework elasticity or Young's modulus, $E$, as probed by single crystal nanoindentation. MOFs have been found to possess highly structure-specific values of $E$, which may be useful in sensing, through mechanical response to external stimuli. ${ }^{9}$ The nature of both the inorganic node and the organic ligand have been shown to affect $E$, which has also been shown to decrease as framework porosity increases (i.e., more open frameworks are also more compliant). ${ }^{10}$ The UiO-66 series of MOFs, ${ }^{11}$ which contain $\mathrm{Zr}_{6} \mathrm{O}_{4}(\mathrm{OH})_{4}$ clusters linked by dicarboxylate ligands, have been

\footnotetext{
${ }^{a}$ WestCHEM, School of Chemistry, The University of Glasgow, University Avenue, Glasgow G12 8QQ, UK. E-mail: Ross.Forgan@glasgow.ac.uk

${ }^{b}$ Department of Materials Science and Metallurgy, University of Cambridge, Cambridge CB3 OFS, UK. E-mail: tdb35@cam.ac.uk

${ }^{c}$ EaStCHEM, School of Chemistry, University of Edinburgh, Joseph Black Building, West Mains Road, Edinburgh, EH9 3JJ, UK

$\dagger$ Electronic supplementary information (ESI) available: Synthesis, bromination, characterisation, crystallography and nanoindentation data. CCDC 1418959-1418961. For ESI and crystallographic data in CIF or other electronic format see DOI: $10.1039 / \mathrm{c} 5 \mathrm{dt} 03178 \mathrm{~h}$
}

predicted to possess significantly larger values of $E$ compared to other highly porous MOFs ${ }^{12}$ linker defects notwithstanding. ${ }^{13}$ These elastic moduli, along with large shear and bulk moduli, have been ascribed to the 12-fold coordination of linkers at the $\mathrm{Zr}^{4+}$ inorganic node, and indicate significant mechanical rigidity for the UiO-66 isoreticular series.

Postsynthetic modification (PSM) ${ }^{14}$ has previously been used to exchange metal or ligand functionalities, ${ }^{15}$ as well as metallate $^{16}$ or covalently modify ${ }^{17}$ organic linkers, within the

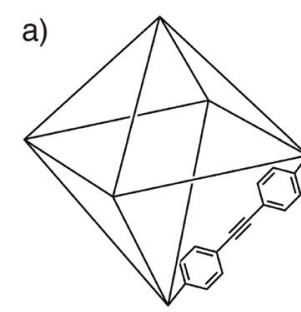

b)
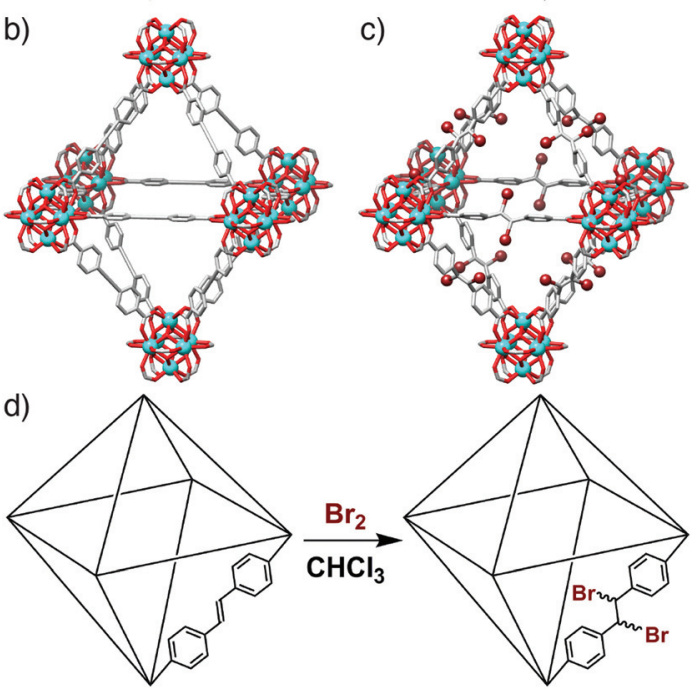

Fig. 1 (a) Schematic of the bromination of (1) to $\left(1-\mathrm{Br}_{2}\right)$. Representations of the single crystal structures of (b) (1), and (c) $\left(1-\mathrm{Br}_{2}\right)$, redrawn from CCDC depositions 1062508 and 1062509 , respectively. ${ }^{18}$ (d) Schematic of the bromination of (2) to $\left(2-\mathrm{Br}_{2}\right)$. 
UiO-66 series. We have recently demonstrated (Fig. 1a-c) that the UiO-type MOF $\left[\mathrm{Zr}_{6} \mathrm{O}_{4}-(\mathrm{OH})_{4}(\mathrm{edb})_{6}\right]_{n}$ (edb $=4,4^{\prime}$-ethynylenedibenzoate), $\$$ denoted henceforth as (1), can undergo quantitative, stereoselective postsynthetic bromination across the integral alkyne units in a single-crystal to single-crystal (SCSC) manner to form (1-Br $\left.{ }_{2}\right)$. This results in a contraction of $4 \%$ in unit cell volume and a change in hybridisation of the linker carbon atoms from sp to $\mathrm{sp}^{2}{ }^{18}$ Herein, we describe the synthesis, characterisation and subsequent SCSC bromination of the analogous alkene containing MOF, $\left[\mathrm{Zr}_{6} \mathrm{O}_{4}(\mathrm{OH})_{4}(\mathrm{sdc})_{6}\right]_{n}$ ( $\operatorname{sdc}=4,4^{\prime}$-stilbene-dicarboxylate, Fig. $\left.1 \mathrm{~d}\right)$, referred to henceforth as (2), including crystallographic characterisation of the brominated material $\left(2-\mathbf{B r}_{2}\right)$. Furthermore, we use single crystal nanoindentation to examine the effect of bromination of both (1) and (2) upon elasticity, or framework compliance.

Bulk samples and single crystals of $(2), \approx 50 \mu \mathrm{m}$ in size and suitable for X-ray diffraction (Fig. 2a), were prepared using our L-proline modulated procedure (see ESI, Section S2 $\dagger$ ). The sdc linker is geometrically frustrated as a result of its non-linearity, leading to end-to-end disorder as seen previously in the crystal structure of $\left(\mathbf{1}-\mathrm{Br}_{2}\right){ }^{18}$ Postsynthetic bromination of alkene units in MOFs has been limited mostly to pendant groups, ${ }^{19}$ with the bromination of a $\mathrm{Zn} \mathrm{MOF}$ containing the sdc linker the only prior example which we are aware of wherein integral alkenes are brominated, although the reaction temperature required to induce quantitative conversion $\left(100{ }^{\circ} \mathrm{C}\right)$ appears detrimental to the crystallinity and porosity of the brominated product. ${ }^{20}$ In contrast, (2) is brominated quantitatively by adding five equivalents of $\mathrm{Br}_{2}$ per alkene unit to a $\mathrm{CHCl}_{3}$ suspension of (2) and soaking for $48 \mathrm{~h}$ in the dark at room temperature (see ESI, Section S3†). NMR spectroscopy of samples of (2) and (2- $\mathrm{Br}_{2}$ ) digested in $\mathrm{D}_{2} \mathrm{SO}_{4} / \mathrm{DMSO}-d_{6}$ was used to ascertain the extent of bromination. Comparison of the ${ }^{1} \mathrm{H}$ NMR spectra of the two digested materials (Fig. 2b) shows complete consumption of the starting material during bromination. The characteristic upfield shift of the resonance assigned to the alkene proton confirms quantitative conversion, with the meso isomer the predominant product. Similar shifts in the signal for the alkene carbon atoms are observed in the ${ }^{13} \mathrm{C}$ NMR spectra (see ESI, Section S4 $\dagger$ ). Minor peaks corresponding to small amounts of a racemic mixture of the diastereomeric products are also visible, unlike the previous report of bromination of a Zn MOF containing the same ligand, which resulted solely in the meso isomer. ${ }^{20}$

The porosities of (2) and (2-Br $\left.{ }_{2}\right)$ were examined by $\mathrm{N}_{2}$ adsorption isotherms carried out at $77 \mathrm{~K}$ (Fig. 2c). BET surface areas decrease from 2900 to $1580 \mathrm{~m}^{2} \mathrm{~g}^{-1}$ upon bromination of (2) in a similar fashion to the values measured for (1) and (1-Br 2 ), 3280 and $2000 \mathrm{~m}^{2} \mathrm{~g}^{-1}$, respectively. ${ }^{18}$ Pore size distributions calculated from the isotherms (see ESI, Section $\mathrm{S} 5 \dagger$ )
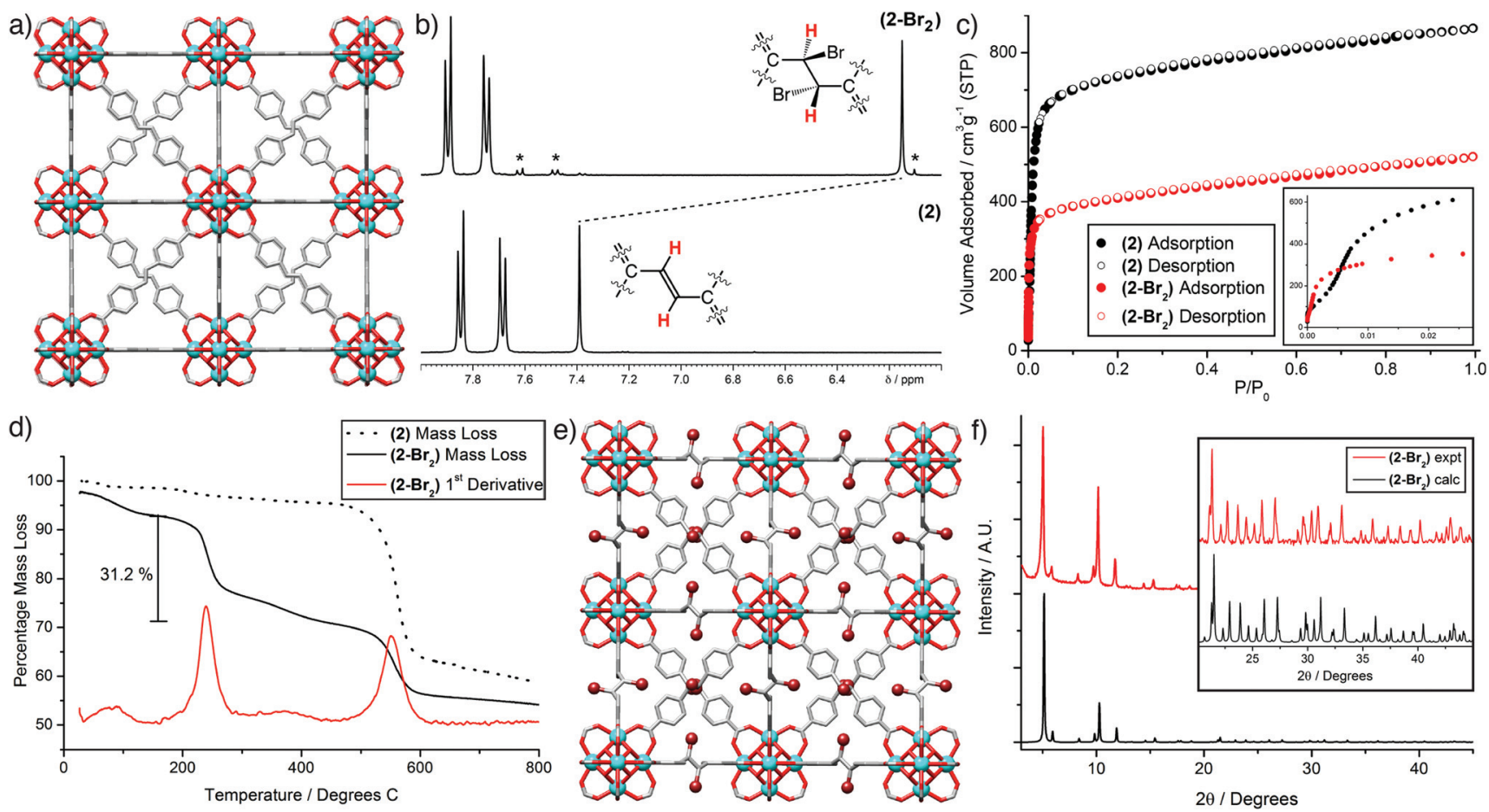

Fig. 2 (a) Representation of the crystal structure of (2) with disorder removed for clarity. (b) Stacked ${ }^{1} \mathrm{H} N M R$ spectra $\left(D_{2} \mathrm{SO}_{4} / \mathrm{DMSO}-d_{6}, 293 \mathrm{~K}\right)$ of digested samples of (2) and (2- $\left.\mathrm{Br}_{2}\right)$, showing the meso isomer is the major product of bromination. Resonances assigned to the racemic mixture of diastereoisomers are marked with an asterix (*). (c) $\mathrm{N}_{2}$ adsorption isotherms collected at $77 \mathrm{~K}$ show a decrease in porosity when (2) is brominated to $\left(2-\mathrm{Br}_{2}\right)$, indicative of a mechanical contraction. (d) Thermogravimetric analysis profiles of (2) and $\left(2-\mathrm{Br}_{2}\right)$ clearly show the effect of bromination on thermal stability. (e) Representation of the crystal structure of $\left(2-\mathrm{Br}_{2}\right)$ with disorder removed for clarity. (f) Powder X-ray diffraction analysis of a bulk sample of $\left(2-\mathrm{Br}_{2}\right)$ compared with the pattern calculated from the crystal structure. 
show a contraction in the main pore diameter from $\approx 11.9 \AA$ for $(2)$ to $\approx 10.1 \AA$ for $\left(2-\mathbf{B r}_{2}\right)$. These values indicate that bromination lowers the surface area and pore volume as a result of mechanical contraction of the MOFs.§

From the thermogravimetric analysis profiles (Fig. 2d), it can be seen that (2) exhibits the typical thermal stability of $\mathrm{Zr}$ MOFs, with the large mass loss around $500{ }^{\circ} \mathrm{C}$ attributed to combustion of the ligand. ${ }^{11}$ In contrast, $\left(2-\mathbf{B r}_{2}\right)$ displays a twostep mass loss profile. Assuming the decrease in mass below $160{ }^{\circ} \mathrm{C}$ is desolvation, then the mass loss in the temperature range $160-450{ }^{\circ} \mathrm{C}$ accounts for $31.2 \%$ of the mass of the desolvated material; the bromine content of fully evacuated (2-Br $\mathbf{2})$ is calculated to be $29.6 \%$. This very close correlation suggests quantitative bromination of (2) has been achieved, supported by a bromine content, determined by elemental analysis, of $27.7 \%$. The possibility of two mass loss mechanisms - debromination and/or elimination of $\mathrm{HBr}$ - may account for the tentative appearance of a very small additional mass loss event around $350-425{ }^{\circ} \mathrm{C}$, but at this point we have been unable to determine whether $\mathrm{Br}_{2}$ or $\mathrm{HBr}$ is being eliminated during the thermal analysis. Elimination of $\mathrm{HBr}$ from the evacuated material would result in a mass loss of $30.0 \%$.

The data collected on the bulk samples all suggest that quantitative bromination has been achieved, and so single crystals of (2), approximately $100 \mu \mathrm{m}$ in size, were subject to similar bromination conditions (see ESI, Section S3†) which allowed the isolation of single crystals of $\left(2-\mathbf{B r}_{2}\right)$. SCSC postsynthetic modification of $\mathrm{MOFs},{ }^{21}$ in particular transformations which alter the linker length or geometry, ${ }^{22}$ are relatively uncommon. As in the crystal structure of the parent material (2) and the related MOF $\left(\mathbf{1}-\mathbf{B r}_{2}\right),{ }^{18}$ the ligand in the solid-state structure of $\left(\mathbf{2}-\mathbf{B r}_{2}\right)$ is geometrically frustrated, and so there is disorder around the central dibromoethane unit. Despite this, the strongly scattering bromine atoms can be clearly observed (Fig. 2e), and the alteration of the ligand geometry is accompanied by a mechanical contraction, with the unit cell edge observed to decrease from 29.8884(3) A for (2) to 29.784(4) $\AA$ for $\left(2-\mathbf{B r}_{2}\right)$ as the hybridisation of the central carbon atoms changes from $\mathrm{sp}^{2}$ to $\mathrm{sp}^{3}$. This contraction is small, but considering the length of the integral $\mathrm{C}-\mathrm{C}$ bond has increased during the transformation, this implies that there is a large amount of flexibility at the centre of the ligand as a consequence of the change in hybridisation. Additionally, the powder X-ray diffraction pattern predicted from the single crystal structure of $\left(2-\mathbf{B r}_{2}\right)$ shows a very close match to the experimental pattern obtained from a bulk brominated sample (Fig. 2f). In concert with the other analytical data described previously, it is clear that the bromination of (2) is quantitative, and results in a highly crystalline, phase pure material with no detrimental effect on porosity.

The availability of single crystals of $\approx 50 \mu \mathrm{m}$ size of all four MOFs enabled nanoindentation to be performed at depths up to $500 \mathrm{~nm}$. The MOFs all display cubic symmetry, and the octahedral crystals were indented on their (111) faces at least 15 times each to derive load-displacement curves (see ESI, Section S6†). Only one facet was indented due to the octahedral nature of the crystals and their small size. The elastic moduli of (1), (1-Br $),(2)$, and (2-Br 2 ), extracted from the raw load-displacement data, show the high values of $E$ characteristic of MOFs of the UiO-66 series (Fig. 3). ${ }^{12}$ In both cases, bromination results in a decrease in the average elastic modulus of the MOF; from $\approx 15.1( \pm 0.8) \mathrm{GPa}$ to $\approx 9.3( \pm 0.6) \mathrm{GPa}$ as $(1)$ is transformed into $\left(1-\mathrm{Br}_{2}\right)$, and from $\approx 11.1( \pm 0.4)$ to $\approx 8.9( \pm 0.4) \mathrm{GPa}$ as $(2)$ is brominated to $\left(2-\mathbf{B r}_{2}\right)$. The decrease in overall porosity and solvent accessible volume associated with bromination of the MOFs would normally be expected to increase the elastic moduli of the materials. ${ }^{10}$

Clearly, the generation of more flexible functionality within the linkers impacts significantly on the materials properties of the MOFs. This hypothesis is supported further by the more porous MOF (1) having a larger value of $E$ than (2), with the only chemical difference between the two being the hybridisation of the carbon atoms of the central bridge of the linker $\left(\mathrm{sp} v s . \mathrm{sp}^{2}\right)$ and the associated increase in degrees of freedom.
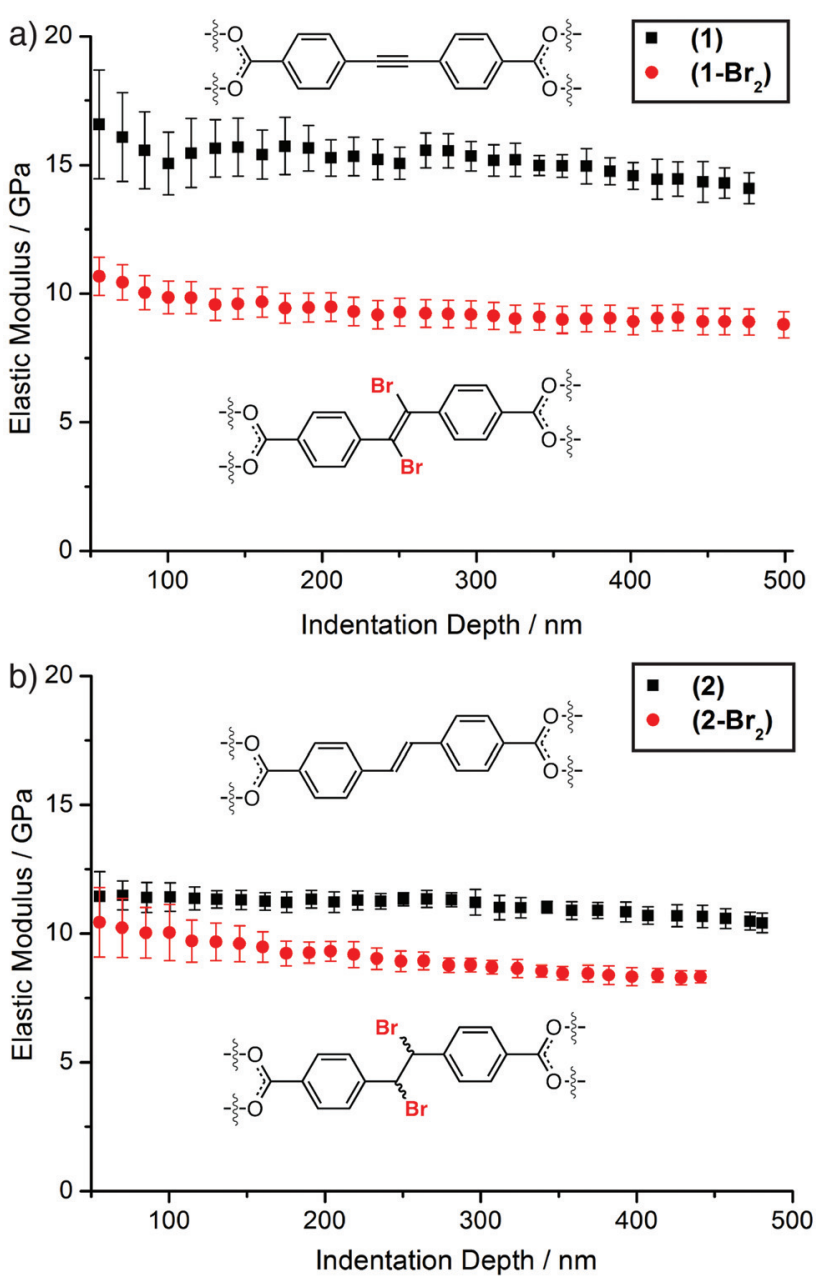

Fig. 3 Comparison of elastic moduli as a function of depth for (a) (1) vs. $\left(1-\mathrm{Br}_{2}\right)$ and (b) (2) vs. $\left(2-\mathrm{Br}_{2}\right)$, showing that bromination results in a reduced elastic modulus in both cases. Error bars are taken from an average of 15 indentations. 


\section{Conclusions}

To conclude, we have demonstrated that postsynthetic modification is a powerful tool to induce changes in mechanical properties of MOFs through changes in ligand functionalisation. Generation of $\mathrm{sp}^{2}$ and $\mathrm{sp}^{3}$ centres, by bromination of unsaturated $\mathrm{C}-\mathrm{C}$ bonds, is facile and quantitative, and the subsequent changes in hybridization significantly impact the elastic moduli of the MOFs. The usefulness of PSM to introduce $\mathrm{sp}^{3}$ hybridised carbons into otherwise rigid MOFs is further demonstrated by the fact that we have been unable as yet to prepare an isoreticular zirconium MOF using the analogous - $\mathrm{CH}_{2} \mathrm{CH}_{2}$ - bridged ligand, 4,4'-ethane-1,2-diyldibenzoic acid (see ESI, Section $\mathrm{S} 7 \dagger$ ), presumably as a result of its conformational freedom. We anticipate that ligand functionalisation will be a powerful tool to modulate mechanical compliance of MOFs in the future.

\section{Acknowledgements}

RSF thanks the Royal Society for receipt of a University Research Fellowship, and the University of Glasgow and EPSRC (EP/L004461/1) for funding. RSF, TDB and SAM gratefully acknowledge pump-priming funding from the EPSRC Directed Assembly Network (PP 1405 03). TDB thanks Trinity Hall (University of Cambridge) for funding. We thank the EPSRC UK National Crystallographic Service for single crystal data collection for $\left(\mathbf{2}-\mathbf{B r}_{\mathbf{2}}\right){ }^{23}$ The data which underpin this work are available at http://dx.doi.org/10.5525/gla.researchdata.231. CCDC 1418959-1418961 contain the supplementary crystallographic data for this paper.

\section{Notes and references}

$\$$ The chemical structures of all ligands and their abbreviation scheme used in this study are given in the ESI, in Scheme S1. $\dagger$

$\S$ The increase in mass of the brominated MOFs is not sufficient to induce the drastic decreases in gravimetric BET surface areas of $\left(\mathbf{1}-\mathbf{B r}_{2}\right)$ and $\left(\mathbf{2}-\mathbf{B r}_{2}\right)$.

1 H. Furukawa, K. E. Cordova, M. O'Keeffe and O. M. Yaghi, Science, 2013, 341, 6149.

2 (a) E. Barea, C. Montoro and J. A. R. Navarro, Chem. Soc. Rev., 2014, 43, 5419-5430; (b) K. Sumida, D. L. Rogow, J. A. Mason, T. M. McDonald, E. D. Bloch, Z. R. Herm, T.-H. Bae and J. R. Long, Chem. Rev., 2012, 112, 724-781.

3 (a) J. Lee, O. K. Farha, J. Roberts, K. A. Scheidt, S. T. Nguyen and J. T. Hupp, Chem. Soc. Rev., 2009, 38, 1450-1459; (b) J. Gascon, A. Corma, F. Kapteijn and F. X. Llabrés i Xamena, ACS Catal., 2014, 4, 361-378.

4 L. E. Kreno, K. Leong, O. K. Farha, M. Allendorf, R. P. Van Duyne and J. T. Hupp, Chem. Rev., 2012, 112, 1105-1125.

5 P. Horcajada, R. Gref, T. Baati, P. K. Allan, G. Maurin, P. Couvreur, G. Férey, R. E. Morris and C. Serre, Chem. Rev., 2012, 112, 1232-1268.
6 A. Schneemann, V. Bon, I. Schwedler, I. Senkovska, S. Kaskel and R. A. Fischer, Chem. Soc. Rev., 2014, 43, 60626096.

7 F.-X. Coudert, Chem. Mater., 2015, 27, 1905-1916.

8 G. Férey and C. Serre, Chem. Soc. Rev., 2009, 38, 1380-1399.

9 J. C. Tan and A. K. Cheetham, Chem. Soc. Rev., 2011, 40, 1059-1080.

10 (a) T. D. Bennett, J.-C. Tan, S. A. Moggach, R. Galvelis, C. Mellot-Draznieks, B. A. Reisner, A. Thirumurugan, D. R. Allan and A. K. Cheetham, Chem. - Eur. J., 2010, 16, 10684-10690; (b) J. C. Tan, T. D. Bennett and A. K. Cheetham, Proc. Natl. Acad. Sci. U. S. A., 2010, 107, 9938-9943.

11 J. H. Cavka, S. Jakobsen, U. Olsbye, N. Guillou, C. Lamberti, S. Bordiga and K. P. Lillerud, J. Am. Chem. Soc., 2008, 130, 13850-13851.

12 H. Wu, T. Yildirim and W. Zhou, J. Phys. Chem. Lett., 2013, 4, 925-930.

13 (a) H. Wu, Y. S. Chua, V. Krungleviciute, M. Tyagi, P. Chen, T. Yildirim and W. Zhou, J. Am. Chem. Soc., 2013, 135, 10525-10532; (b) M. J. Cliffe, W. Wan, X. Zou, P. A. Chater, A. K. Kleppe, M. G. Tucker, H. Wilhelm, N. P. Funnell, F.-X. Coudert and A. L. Goodwin, Nat. Commun., 2014, 5, 4176; (c) B. Van de Voorde, I. Stassen, B. Bueken, F. Vermoortele, D. De Vos, R. Ameloot, J.-C. Tan and T. D. Bennett, J. Mater. Chem. A, 2015, 3, 1737-1742.

14 S. M. Cohen, Chem. Rev., 2012, 112, 970-1000.

15 (a) M. Kim, J. F. Cahill, H. Fei, K. A. Prather and S. M. Cohen, J. Am. Chem. Soc., 2012, 134, 18082-18088; (b) H. Fei and S. M. Cohen, J. Am. Chem. Soc., 2015, 137, 2191-2194.

16 (a) M. I. Gonzalez, E. D. Bloch, J. A. Mason, S. J. Teat and J. R. Long, Inorg. Chem., 2015, 54, 2995-3005; (b) K. Manna, T. Zhang, F. X. Greene and W. Lin, J. Am. Chem. Soc., 2015, 137, 2665-2673.

17 (a) S. J. Garibay and S. M. Cohen, Chem. Commun., 2010, 46, 7700-7702; (b) M. Kandiah, S. Usseglio, S. Svelle, U. Olsbye, K. P. Lillerud and M. Tilset, J. Mater. Chem., 2010, 20, 9848-9851; (c) W. Morris, W. E. Briley, E. Auyeung, M. D. Cabezas and C. A. Mirkin, J. Am. Chem. Soc., 2014, 136, 7261-7264.

18 R. J. Marshall, S. L. Griffin, C. Wilson and R. S. Forgan, J. Am. Chem. Soc., 2015, 137, 9527-9530.

19 (a) K. Hindelang, A. Kronast, S. I. Vagin and B. Rieger, Chem. - Eur. J., 2013, 19, 8244-8252; (b) T. K. Prasad and M. P. Suh, Chem. - Eur. J., 2012, 18, 8673-8680; (c) F. Sun, Z. Yin, Q.-Q. Wang, D. Sun, M.-H. Zeng and M. Kurmoo, Angew. Chem., Int. Ed., 2013, 52, 4538-4543; (d) Z. Wang and S. M. Cohen, Angew. Chem., Int. Ed., 2008, 47, 4699-4702.

20 S. C. Jones and C. A. Bauer, J. Am. Chem. Soc., 2009, 131, 12516-12517.

21 J.-P. Zhang, P.-Q. Liao, H.-L. Zhou, R.-B. Lin and X.-M. Chen, Chem. Soc. Rev., 2014, 43, 5789-5814.

22 P.-Q. Liao, A.-X. Zhu, W.-X. Zhang, J.-P. Zhang and X.-M. Chen, Nat. Commun., 2015, 6, 6350.

23 S. J. Coles and P. A. Gale, Chem. Sci., 2012, 3, 683-689. 